depression, and that dosage can be increased within the range 20 to $60 \mathrm{mg}$ without risk of increasing SSRI-type side effects.

\section{Tues-P25 \\ CITALOPRAM INFUSION IS A USEFUL ALTERNATIVE TO TABLETS IN HOSPITALISED PATIENTS WITH DEPRESSION}

P. Baumann ${ }^{1}, \mathrm{R} . \mathrm{Nil}^{2 *}$. 'Departement Universitaire de Psychiatrie Adulte, Prilly, Lausanne, Switzerland

${ }^{2} H$. Lundbeck A/S, Copenhagen, Denmark

It has been suggested that it can be advantageous to use intravenous (IV), rather than oral antidepressants in severely depressed patients. The IV route avoids first-pass metabolism of the drug, and may result in a faster onset of action; infusion may also have a psychotherapeutic effect. Few of the second-generation antidepressants are available for IV therapy, and citalopram is the only selective serotonin re-uptake inhibitor that has been formulated for infusion. Citalopram is highly efficacious and has a good safety profile. Its bioavailability is high, and its metabolites are of little clinical significance; therefore, oral and IV doses are equivalent. This randomised, parallel-group study compared citalopram tablets with citalopram two-hour infusion, each given once daily in a double-blind, double-dummy design, followed by open-label oral citalopram. Sixty patients (mean age 43 years), hospitalised for moderately - severe depression, received either citalopram tablet plus placebo infusion $(n=30)$, or placebo tablet plus citalopram infusion $(n=30)$ for 10 days. All patients then received open treatment with oral citalopram (Days 11-42). The daily dosage in both groups was $20 \mathrm{mg}$ on Days 1-2, $40 \mathrm{mg}$ on Days 3-14, and $60 \mathrm{mg}$ on Days $15-21$, reducing thereafter to $40 \mathrm{mg}$ if clinically indicated. On Day 7, the reduction from baseline on the Hamilton Depression (HAMD) Scale was numerically greater in the infusion group than in the tablet group (6.3 vs 4.3 ; NS) suggesting a more rapid onset of effect with infusion. This trend was also apparent on Day 11 , when $50 \%$ and $37 \%$ of patients in the infusion and tablet groups, respectively, were classed as responders on the Clinical Global Impression (CGI) scale. On Day 42, the proportion of responders in the two groups was identical $(73 \%)$, and the decrease from baseline in HAMD and CGI was significant in both groups (p $<0.001)$. There were no clinically relevant differences in adverse events or safety variables between the groups. These results suggest that slow-drop infusion of citalopram has a similar risk/benefit ratio, and may have a more rapid onset of antidepressant effect, than oral citalopram.

\section{Tues-P26}

BENEFITS OF CITALOPRAM VS VILOXAZINE, BOTH GIVEN AS AN INTRAVENOUS-TO-ORAL REGIMEN FOR SEVERE DEPRESSION

J.M. Bouchard', R. Nil' *. 'C.G.S. Gérard Marchant, Toulouse, France

${ }^{2} H$. Lundbeck A/S, Copenhagen, Denmark

There is anecdotal evidence that antidepressant effects are observed more quickly if the drug is administered intravenously (IV) rather than orally. In addition, compliance is assured by IV therapy. Therefore, commencing treatment with an infusion appears beneficial for severely depressed patients. Most available IV antidepressants are tricyclics (TCAs) or tetracyclics, and their use is limited by an unfavourable side effect profile, particularly cardiotoxicity. Selective serotonin re-uptake inhibitors (SSRIs) are as efficacious as TCAs but have a better safety profile. Citalopram is the most selective SSRI and the only one available as an infusion. This randomised, double-blind, parallel-group, multicentre study, conducted in France, compared the efficacy and safety of citalopram with viloxazine, each administered by slow-drop infusion for 2 weeks, then orally for 4 weeks, in 65 patients (aged 23-70 years) hospitalised for major depression. Patients received either citalopram ( $40 \mathrm{mg} /$ day IV then orally; $n=32$ ), or viloxazine (300 mg/day IV then $600 \mathrm{mg} /$ day orally; $n=33$ ). There were 11 withdrawals from each treatment group, the most common reasons for these being 'improvement' in the citalopram group, and 'lack of efficacy' in the viloxazine group. The mean Montgomery-Asberg Depression Rating Scale total score was 34 in both treatment groups at baseline. After treatment, this score was significantly $(p<0.05)$ lower in the citalopram group than in the viloxazine group, both on Day 14 (12.3 us 16.9) and Day 42 (6.7 us 13.1). Improvement in Clinical Global Impression scores was also significantly ( $p<0.015$ ) greater in the citalopram group (Day 42). Treatment-emergent nausea and constipation occurred most frequently in the viloxazine group, whereas weight gain and concentration difficulties were more frequent with citalopram. No clinically significant cardiac events occurred in either group, and injection site tolerability was good with both drugs. In conclusion, an IV/oral regimen of citalopram is more efficacious than a similar regimen of viloxazine in patients with severe depression.

\section{Tues-P27 \\ TOLERABILITY OF 15 VS 30 MG INITIAL DOSES OF MIR- TAZAPINE: A RANDOMIZED, DOUBLE-BLIND STUDY}

J.T.H. Helsdingen *, M. Zivkov. NV Organon, Molenstraat 110, BH 5340 Oss, The Netherlands

Aim: To assess the tolerability of 2 different initial doses of mirtazapine, outpatients with a DSM IV diagnosis of a Major Depressive Episode were randomly assigned to an ascending dosage regimen $(n=71$; mirtazapine $15 \mathrm{mg}$ for 1 week, followed by 30 $\mathrm{mg}$ for 1 week) or a fixed dosage regimen ( $\mathrm{n}=69,30 \mathrm{mg}$ for 2 weeks).

Methods: Tolerability was assessed by recording of adverse events (AEs), and using the computer-assisted interactive telephone system for daily ratings on the VAMRS scale, with 'Alert/drowsy' factor as an index of a day-time sedation. Efficacy was assessed by the17-HAMD and CGI, and effects on sleep by self ratings on the LSEQ, using the same computer-assisted system.

Results: Tolerability of both treatments was good. A total of 3 patients in each treatment group dropped-out; respectively 1 and 2 patients because of adverse events. During the first treatment week, AEs were reported with a similar incidence in both groups: somnolence by $9.9 \%$ of patients in the $15 \mathrm{mg}$ group, and by $10.1 \%$ in the $30 \mathrm{mg}$ group; respective values for dizziness were $4.2 \%$ and $8.7 \%$. On the 'Alert/drowsy' factor a similar level of a daytime sedation was registered in both groups after the first dose of study medication, with subsequent immediate increase in alertness to baseline values, and approx. at day 10, to the level of 'normal' state. In both groups 17-HAMD scores decreased similarely at endpoint $(-9.5 \pm 5.9$ and $-10.9 \pm 6.5)$. On the LSEQ, $30 \mathrm{mg}$ initial dose of mirtazapine was related to a statistically significantly longer duration of sleep at weeks 1 and 2 , and to significantly faster initiation of sleep at week 2 .

Conclusion: There are no differences in tolerability of mirtazapine administered in initial doses of 15 or $30 \mathrm{mg}$, and both dosage regimens are well tolerated. The results on LSEQ were in favor of $30 \mathrm{mg}$ initial dose, with respect to onset and duration of sleep. 